\title{
Review of: "Polycomb group protein CBX7 represses cardiomyocyte proliferation via modulation of the TARDBP/Rbm38 axis"
}

\author{
Liansheng Wang ${ }^{1}$ \\ 1 Nanjing Medical University
}

Potential competing interests: The author(s) declared that no potential competing interests exist.

The authors aim to explore the anti-proliferation effects of Polycomb group protein CBX7 in animal and cell models. They demonstrated that CBX7 inhibits the proliferation of perinatal CMs by controlling TARDBP/Rbm38 pathway. This is an interesting study. Because, in most of the previous studies, researchers pay more attention to the changes in proliferative capacity from newborn to adult. In this study, the authors explore the role of $\mathrm{CBX7}$ in $\mathrm{CM}$ proliferation from the prenatal to the postnatal stage. And, through immunoprecipitation and mass spectrometry(MS), they further uncovered a mechanism regulating CM proliferation via CBX7- TARDBP axis. The authors can address the following comments and concerns to improve the manuscript.

1. The assays utilized to assess CM proliferation are not state of the art. There is no formal demonstration of cardiomyocyte cell division in this analysis. This does not detract substantially from the work, but the authors too easily conclude cell division when this was not shown. Expression of more cell proliferation markers(e.g., EdU and Aurora Kinase B [Aurora B]) are necessary to be analyzed. Reporter mice are also a good choice to permanently mark cardiomyocytes and follow the fate of their cellular descendants in vivo.

2. There are some concerns about data quality. The authors concluded that $\mathrm{CBX} 7$ is involved in cardiomyocyte proliferation mainly based on the expression of the marker genes in immunofluorescent staining. Ki67 and pH3 positive CMs should be indicated by the arrows. It would be more convincing to use PCM1 as a CM marker and detect the co-localization of PCM1 and proliferation markers.

3. We find that, to explore the underlying mechanism, authors investigated potential cytoplasmic binding partners of CBX7 through immunoprecipitation in MEFs. The results need to be confirmed in CMs. Because this study focuses on the effect of CBX7 in CM proliferation.

4. The author can supplement echocardiography or cardiac Magnetic Resonance Imaging to clarify the influence of CBX7 overexpression or inhibition on the cardiac function when conducting the gain-and-loss function analyses in vivo, and it is also a good supplement to Figure2 D-F to prove the critical role of CBX7 in cardiac development.

5. In Figure 1 and S2, does Ad-CBX7 specially enhance CBX7 expression in CMs? If not, how do authors exclude the possibilities that CBX7 over-expression inhibited CM proliferation by targeting non-CMs.

6. CBX7 has been suggested to regulate cell proliferation mostly in cancer. However, studies reported 
opposite functions of CBX7 in cellular proliferation as an oncogene or a tumor suppressor. In summary, authors concluded that the role of CBX7 could be tissue-specific and context-specific. Therefore, the author should focus on the underlying mechanism of this controversial effect or put forward his conjecture in the discussion section. 